\title{
Inhibitory effect of Lycium barbarum polysaccharide on sperm damage during cryopreservation
}

\author{
BEI YAN $^{1 *}$, XINZONG ZHANG ${ }^{2 *}$, JUAN WANG $^{3}$, SHAOTONG JIA ${ }^{4}$, \\ YUE ZHOU $^{1}$, JIA TIAN ${ }^{1}$, HONGYAN WANG $^{1}$ and YUNGE TANG ${ }^{2}$ \\ ${ }^{1}$ Ningxia Human Sperm Bank, General Hospital of Ningxia Medical University, Yinchuan, Ningxia 750001; \\ ${ }^{2}$ National Health Committee Key Laboratory of Male Reproduction and Genetics, Family Planning Research \\ Institute of Guangdong Province, Guangzhou, Guangdong 510600; ${ }^{3}$ Department of Pathology, \\ Ningxia Medical University, Yinchuan, Ningxia 750004; ${ }^{4}$ Reproductive Center, General \\ Hospital of Ningxia Medical University, Yinchuan, Ningxia 750001, P.R. China
}

Received December 5, 2019; Accepted May 29, 2020

DOI: $10.3892 /$ etm.2020.9060

\begin{abstract}
With the development of cryopreservation technology, marked progress has been made regarding sperm cryopreservation. However, as conventional cryopreservation agents are not effective at freezing weak sperm, improved cryopreservation agents are in demand. In the present study, the addition of Lycium barbarum polysaccharides to glycerol-egg-yolk-citrate sperm cryopreservation agent was determined to improve sperm forward speed, reduce the DNA fragmentation index and improve the mitochondrial membrane potential. Furthermore, during the freezing and thawing of sperm, the improved cryopreservative increased the content of Bcl-2 while reducing the content of Bax, cytochrome $\mathrm{C}$ and caspase- 3 . These results indicated that polysaccharides added as a protective agent preserved the normal function of sperm mitochondria. Transmission electron microscopy also confirmed the protective effect of the polysaccharides on the structure of mitochondria. It was also indicated that improved cryopreservative lowered the levels of reactive oxygen species (ROS) during the freeze-thawing process. Therefore, it is hypothesized that improved cryopreservative agents may be beneficial for maintaining the structure and function of
\end{abstract}

Correspondence to: Professor Yunge Tang, National Health Committee Key Laboratory of Male Reproduction and Genetics, Family Planning Research Institute of Guangdong Province, 17 Meidong Road, Yuexiu, Guangzhou, Guangdong 510600, P.R. China E-mail:tyg813@126.com

Professor Hongyan Wang, Ningxia Human Sperm Bank, General Hospital of Ningxia Medical University, 804 Shengli South Street, Xingqing, Yinchuan, Ningxia 750001, P.R. China

E-mail: 1099382469@qq.com

*Contributed equally

Key words: ultralow temperature, sperm cryopreservation, mitochondria, reactive oxygen species the mitochondria of weak sperm when cryopreserved, which may be facilitated via reducing the production of ROS in the freezing-thawing process, thus avoiding activation of the apoptotic pathway in sperm mitochondria and protecting mitochondrial structure and sperm function.

\section{Introduction}

Sperm cryopreservation is not only a routine part of the daily work performed at sperm banks but also an indispensable part of assisted reproductive technology. Ultralow temperatures may lead to impaired sperm motility and the formation of ice crystals during freezing and thawing, as well as excessive oxidative stress during this process that may damage the structure and function of sperm $(1,2)$. According to the World Health Organization (WHO), $50 \%$ of sperm cells are damaged or killed during thawing (3). The sperm is mixed with cryopreservation agents prior to freezing in order to reduce its mortality (4). The most commonly used cryopreservation agent is glycerol-egg-yolk-citrate (GEYC) cryopreservative (5). However, conventional cryopreservation agents are not sufficiently able to preserve weak sperm (2). In recent years, domestic and international studies have reported on the addition of natural or synthetic substances to cryopreservation agents to reduce the freezing-associated damage of sperm (2,6-9). Shahrzad and other researchers (10-12) indicated that the addition of L-carnitine to cryopreservative may effectively lower the sperm DNA fragmentation index and apoptosis. Li et al (13) indicated that the addition of $200 \mathrm{nmol} / 1$ mitochondria-targeted antioxidant mitoquinone (MitoQ) to cryopreservative may to markedly improve the quality of cryopreserved sperm, and it was also proposed that MitoQ could also be used as a cryopreservation additive for semen. Berkovitz et al (14) indicated that $\mathrm{Zn}^{2+}$ may also improve the motility of cryopreserved sperm cells. An increasing number of studies have also made efforts to identify natural protective agents that are able to improve the motility of cryopreserved sperm $(2,6,7)$.

Lycium barbarum, also known as 'Oriental magic grass', is commonly used in the Ningxia Hui Autonomous Region 
of northwest China, as a traditional Chinese medicine to treat male infertility, hypertension, high cholesterol and diabetes (15). In recent years, Lycium barbarum polysaccharides (LBP) extracted from this plant have been used as antioxidants, scavenging oxygen free radicals and stabilizing cell membranes (16-20). A previous study indicated that the addition of LBP during semen treatment was able to protect sperm structure and function (21). Therefore, in the present study, LBP was added into the GEYC cryopreservative to investigate its effect on the structure and function of mitochondria of weak sperm, to provide a theoretical basis for further research into cryopreservation agents that may be beneficial in maintaining the function of cryopreserved sperm. Sperm motility requires energy derived from the ATP produced by mitochondria. Therefore, the structure and function of mitochondria after freezing are crucial to sperm motility (9). During the sperm freeze-thawing process, a considerable amount of reactive oxygen species (ROS), which can damage the mitochondrial membrane, are produced, resulting in a significant decrease in sperm motility $(1,2)$. In the present study, the four key regulators of mitochondrial apoptosis pathways, including Bcl-2, Bax, cytochrome C (CytC) and caspase-3 (22), were assessed as indicators for mitochondrial apoptosis pathway activation. The effects of LBP on the freeze-thawing of weak sperm and on the key molecules in these apoptosis pathways, were studied by measuring the level of ROS. The aim was to further explore the protective mechanism of improved cryopreservative and to identify more effective protective agents that can be used in sperm cryopreservation.

\section{Materials and methods}

Sample collection and selection criteria. Between May and June 2018, 22 patients with asthenospermia, aged 22-45 (mean age $31.9 \pm 8.8$ ) years and with a semen volume of $2.4 \pm 0.6 \mathrm{ml}$ were selected from the reproductive center of Ningxia Medical University General Hospital. The selection criteria were in accordance with the WHO Human Semen Examination and Processing Laboratory Manual (Fifth Edition) (23) and were as follows: Semen volume, $\geq 1.5 \mathrm{ml}$; total sperm number, $\geq 39$ $\left(10^{6} / \mathrm{ml}\right)$; sperm forward progressive motility (PR) $<32 \%$; sperm $[\mathrm{PR}+$ non-progressive motility $(\mathrm{NP})]<40 \%$. Samples with a sperm concentration range of $6-7 \times 10^{7} / \mathrm{ml}$ were used in further experiments. In order to eliminate dysliquefaction and severe oligospermia, peroxidase staining was used to eliminate leukocytospermia (peroxidase-positive leukocytes; $<1.0 \times 10^{6} / \mathrm{ml}$ ). To perform peroxidase staining $125 \mathrm{mg}$ benzidine was dissolved in $50 \mathrm{ml}$ 95\% methanol. A $150 \mathrm{mg}$ quantity of rose red $\mathrm{B}$ was dissolved in $50 \mathrm{ml}$ of distilled water and the two solutions were mixed. A $1 \mathrm{ml}$ volume of this mixed solution had 2 drops of $\mathrm{H}_{2} \mathrm{O}_{2}$ added and the prepared solution formed the benzidine dye. A drop of semen was placed on a slide with a drop of benzidine dye solution and mixed well. The slide was covered, placed at $37^{\circ} \mathrm{C}$ for $20 \mathrm{~min}$ and observed under a light microscope $(\mathrm{x} 1,000)$. White blood cells were brown after staining. The number of leukocytes were observed in 200 sperm visual fields. The present study was approved by the ethics committee of the General Hospital of Ningxia Medical University (Yinchuan, China) and written informed consent was obtained from the patients.
Sample preparation. All the subjects were abstinent for 3-5 days. The semen samples were collected by masturbation into aseptic seminal cups. Each semen sample was first placed in a $37^{\circ} \mathrm{C}$ water bath for $30 \mathrm{~min}$. After the sample was completely liquefied and evenly mixed, 1,500 $\mu \mathrm{l}$ of each semen sample was divided into three groups: Fresh group (unfrozen sperm), GEYC group (frozen sperm with standard protectant) and LBP group (frozen group with standard protectant and added LBP; $500 \mu \mathrm{l}$ semen in each group). After liquefication, the semen was diluted with $500 \mu \mathrm{l}$ Biggers, Whitten and Whittingham (BWW) sperm culture solution (Beijing Solarbio Science \& Technology Co., Ltd.; cat. no. G2586), as recommended by the WHO guidelines (23), and centrifuged at $500 \mathrm{x} \mathrm{g}$ for $10 \mathrm{~min}$ at room temperature. The supernatant was then discarded, and $1 \mathrm{ml}$ sperm culture solution was added (Fresh: $1 \mathrm{ml} \mathrm{BWW}$; GEYC: $1 \mathrm{ml}$ BWW, LBP: $1 \mathrm{ml} \mathrm{BWW}$ containing 1,000 $\mu \mathrm{g} / \mathrm{ml}$ LBP), followed by incubation at $37^{\circ} \mathrm{C}$ for $20 \mathrm{~min}$.

The sperm was then frozen; the cryoprotectant base fluid was prepared according to the protocol for the GEYC protectant recommended by the WHO guidelines (23).

Cryopreservation and cryo-survival of sperm. The manual two-step freezing method was employed (9). Semen and protectant were added to the cryopreservation tube at a ratio of 2:1 and the cryopreservation tube was placed at a height of $\sim 5 \mathrm{~cm}$ from the liquid nitrogen surface for $10 \mathrm{~min}$. Subsequently, it was lowered to $0.1 \mathrm{~cm}$ above the liquid level (no contact with the liquid level) for $5 \mathrm{~min}$ and subsequently immersed in liquid nitrogen for preservation.

Sperm cryo-survival. The cryopreservation tube was taken out of the liquid nitrogen and placed in a $37^{\circ} \mathrm{C}$ water bath for rewarming for $3 \mathrm{~min}$. The sample was then centrifuged $300 \mathrm{x} \mathrm{g}$ for $5 \mathrm{~min}$ at room temperature and placed into the BWW culture medium for $10 \mathrm{~min}$ at $37^{\circ} \mathrm{C}$. Subsequently, the suspension was gently reversed 10 times and evenly mixed and then sperm was then subsequently tested for viability.

Detection of sperm-associated activity parameters. Computer-aided sperm analysis technology (CASA) was used to analyse the relevant parameters. Prior to the assessment of sperm viability, the counting plate [Sefi-Medical Instruments (9), Ltd.] was placed on the temperature control plate of the microscope in advance. This was a single chamber system. A total of six representative fields were examined under light microscopy (x200), at least 200 spermatozoa were assessed for each sample. The test was repeated twice. The color sperm quality detection system (WLJY-9DOO; version 2.0; Beijing Weili New Century Science \& Tech. Deve. Co., Ltd.) was used to determine the sperm vitality [percentage of sperm forward movement (PR)] and other relevant parameters [curvilinear velocity (VCL), straight linear velocity (VSL), sperm average path velocity (VAP), amplitude of lateral head displacement (ALH) and beat cross frequency (BCF)] sperm concentration was $2-50 \times 10^{6}$. Fast forward motion with a speed $\geq 25 \mu \mathrm{m} / \mathrm{sec}$ was classified as class $\mathrm{A}$; if the velocity of forward movement was $<25 \mu \mathrm{m} / \mathrm{sec}$, it was classified as class $\mathrm{B}(\mathrm{PR}=\mathrm{A}+\mathrm{B})$; if the velocity of non-forward movement or forward movement was $\leq 5 \mu \mathrm{m} / \mathrm{S}$, it was classified as class $\mathrm{C}(\mathrm{NP}=\mathrm{C})$; Total immobility was classified as level D (immobility=D). The spermatozoa 
were incubated $37^{\circ} \mathrm{C}$ for 10 min prior to measuring motility. If the sperm concentration was $>50 \times 10^{6}$, considering the high frequency of sperm collision producing errors, the semen was diluted with BWW to $2-50 \times 10^{6}$ before assessment.

Sperm survival rate experiment. The survival rate was assessed using eosin aniline black (Beijing Solarbio Science \& Technology, Co., Ltd.; cat. no. G2581), as recommended by the WHO guidelines (23). The sperm concentration was adjusted to $2-50 \times 10^{6}$ using normal saline and $25 \mu \mathrm{l}$ of semen and an equal volume of eosin aniline black suspension were added to a $1.5 \mathrm{ml}$ centrifuge tube, mixed with micropipette at room temperature and left to stand for $30 \mathrm{sec}$. A sperm smear was made with each sperm suspension, dried and checked immediately after drying. Each glass slide was examined using a light-field microscope under an oil immersion objective (magnification, $x 1,000$ ). Finally, with the help of a sperm counter (cat. no. XK06-6J; Jiangsu Xinkang Medical Devices, Co., Ltd.), the stained and non-stained sperm were counted. Each sample was evaluated twice and 200 sperm from each sample were evaluated to achieve an acceptably low sampling error. If the difference was too large, re-sampling and re-evaluation were performed. Fig. 1 presents the evaluation criteria used.

Sperm morphology test. The sperm morphology was observed using a diff Quik rapid staining kit (cat. no. G1541; Beijing Solarbio Science \& Technology Co., Ltd.) First, the sperm concentration was adjusted to $\left(2-50 \times 10^{6}\right)$ using normal saline, and a sperm smear was then prepared and immersed in fast dye solution I at room temperature for $10 \mathrm{sec}$, followed by fast dye solution II at room temperature for $5 \mathrm{sec}$. The slide was then rinsed under running water to remove excess dye solution. A total of 200 sperms were evaluated after sealing under a light microscope $(\mathrm{x} 1,000)$. The numbers of normal and abnormal spermatozoa were recorded, and the percentage was calculated. This staining method is recommended by the WHO laboratory manual for human semen examination and processing (Fifth Edition) (23).

\section{Sperm function tests}

DNA fragment index (DFI) test. The DFI of sperm was assessed using a sperm chromatin structure assay (SCSA) (24). A DFI cell flow cytometer test kit (cat. no. CP0101-10T; Cellpro; Zhejiang Xingbo Biological Technology Co., Ltd.) was selected for testing; this kit has been approved by the State Food and Drug Administration (SFDA) for clinical semen examination (approval no. 20160051). Solution A contained: $0.1 \mathrm{~mol} / 1 \mathrm{NaCl}, 0.4 \mathrm{mmol} / 1 \mathrm{KH}_{2} \mathrm{PO}_{4}, 2.7 \mathrm{mmol} / \mathrm{l}$ $\mathrm{C}_{6} \mathrm{H}_{6} \mathrm{O}_{6}, 4.7 \mathrm{mmol} / 1 \mathrm{KCl}, 0.2 \mathrm{mmol} / 1 \mathrm{MgSO}_{4} \cdot 7 \mathrm{H}_{2} \mathrm{O}, 2 \mathrm{mmol} / 1$ $\mathrm{CaCl}_{2} \cdot 2 \mathrm{H}_{2} \mathrm{O}, 0.02 \mathrm{~mol} / 1 \mathrm{HEPES}-\mathrm{Na}, 4 \mathrm{mmol} / 1 \mathrm{NaHCO}_{3}$, $0.3 \mathrm{mmol} / 1 \mathrm{C}_{3} \mathrm{H}_{3} \mathrm{O}_{3} \mathrm{Na}, 0.34 \%$ (v/v) $\mathrm{C}_{3} \mathrm{H}_{5} \mathrm{O}_{3} \mathrm{Na}, 40.5 \%$ (v/v) Percol. Solution B contained $0.1 \mathrm{~mol} / 1 \mathrm{NaCl}, 0.4 \mathrm{mmol} / 1$ $\mathrm{KH}_{2} \mathrm{PO}_{4}, 2.7 \mathrm{mmol} / 1 \mathrm{C}_{6} \mathrm{H}_{6} \mathrm{O}_{6}, 4.7 \mathrm{mmol} / 1 \mathrm{KCl}, 0.2 \mathrm{mmol} / \mathrm{l}$ $\mathrm{MgSO}_{4} \bullet 7 \mathrm{H}_{2} \mathrm{O}, 2 \mathrm{mmol} / 1 \mathrm{CaCl}_{2} \bullet 2 \mathrm{H}_{2} \mathrm{O}, 0.02 \mathrm{~mol} / 1 \mathrm{HEPES}-\mathrm{Na}$, $4 \mathrm{mmol} / 1 \mathrm{NaHCO}_{3}, 0.3 \mathrm{mmol} / 1 \mathrm{C}_{3} \mathrm{H}_{3} \mathrm{O}_{3} \mathrm{Na}, 81 \%$ (v/v) Percol. $1 \%$ (v/v) Triton X-100 (Beijing Solarbio Science \& Technology Co., Ltd.; T8200); Solution C contained $6 \mu \mathrm{g} / \mathrm{ml}$ acridine orange, $37 \mathrm{mmol} / 1$ citric acid, $126 \mathrm{mmol} / 1 \mathrm{Na}_{2} \mathrm{HPO}_{4}, 1 \mathrm{mmol} / 1$ disodium EDTA, $0.15 \mathrm{~mol} / \mathrm{l} \mathrm{NaCl}$. The specific operation was as follows: Sperm $1-2 \times 10^{6} / \mathrm{ml}$ was diluted with solution A to a final volume of $100 \mu \mathrm{l}$. Subsequently, $200 \mu \mathrm{l}$ of solution B was added, followed by incubation at room temperature for $30 \mathrm{sec}$ (accurate timing was required). A total of $600 \mu \mathrm{l}$ solution $\mathrm{C}$ was added and a BD accuri C6 (BD Biosciences) flow cytometer was used to select the cell groups to be studied on the working interface. The sperm flow rate was controlled at $100-300 / \mathrm{sec}$ and after two minutes of stable operation, at least 5,000 sperm were collected, and each sample was assessed at least twice. After data collection, the flow cytometry (FCS) files were copied into DFI-View software (DFIView; version 1.10; Cellpro; Zhejiang Xingbo Biological Technology Co., Ltd.) for analysis.

Sperm mitochondrial membrane potential (MMP) test. The sperm tail interruption staining kit (cat. no. CP01014-10T; Cellpro; Zhejiang Xingbo Biological Technology Co., Ltd.) was used for the detection of MMP. The kit has been approved by the SFDA for clinical semen examination (zyxb no. 20160052). The specific operation was as follows: The semen was diluted to $2 \times 10^{6} / \mathrm{ml}$ and $0.5 \mathrm{ml} \mathrm{JC}-1$ working solution was added, followed by incubation at $37^{\circ} \mathrm{C}$ for $20 \mathrm{~min}$ and centrifugation at $4^{\circ} \mathrm{C}$ and $300 \times \mathrm{g}$ for $5 \mathrm{~min}$. The supernatant was removed, and the precipitated cells were washed twice with JC-1 buffer solution, and the cells were analyzed using a BD Accuri C6 (BD Biosciences) with an excitation wavelength of $488 \mathrm{~nm}$ and emission wavelength was $530 \mathrm{~nm}$. The sperm flow rate was controlled at $100-300 / \mathrm{sec}$, and at least 5,000 sperm were collected, and each sample was detected at least twice. Following data collection, the FCS files were copied into MMP-test software (MMP-Test; Version 1.0; Cellpro; Zhejiang Xingbo Biological Technology Co., Ltd.) for analysis. For sperm with a high MMP, JC-1 spontaneously forms JC-1 aggregates with intense red fluorescence, which is measured on the FL2 channel. For sperm with low MMP, JC-1 remains in the monomer form and exhibits green fluorescence, which is measured on the FL1 channel (25).

Acrosome reaction (AR) of sperm test. The Sperm Acrosome Staining kit (acrosome test; cat. no. CP01011-10T; Cellpro; Zhejiang Xingbo Biological Technology Co., Ltd.) was selected for detection. The kit has been approved by the SFDA for clinical semen examination (approval no. 20160054). The specific operation was as follows: The sperm concentration was adjusted to $4-5 \times 10^{6}$ live sperm using solution $\mathrm{A}$; two $1.5-\mathrm{ml}$ centrifuge tubes were prepared and $10 \mu \mathrm{l}$ solution $\mathrm{B}$ and $10 \mu \mathrm{l}$ solution $\mathrm{C}$ was added. The sample was then mixed and incubated at $37^{\circ} \mathrm{C}$ for $15 \mathrm{~min}$, and $100 \mu \mathrm{l}$ pre-cooled $70 \%$ ethanol was added into each tube, followed by incubation for $2 \mathrm{~min}$ at room temperature. The mixture was centrifuged at $500 \mathrm{xg}$ for $5 \mathrm{~min}$ at room temperature, the supernatant was discarded and $500 \mu \mathrm{l}$ staining working solution (mixture of solution D and solution E) was added, followed by shaking and mixing. Subsequently, the mixture was incubated for $15 \mathrm{~min}$ at $37^{\circ} \mathrm{C}$. On the BD Accuri C6 equipment the voltage of the FL1 channel was adjusted so that the major group was in the specified gates. The sperm flow rate was controlled at $100-300 / \mathrm{sec}$, and at least 5,000 sperm were collected each sample was analyzed at least twice. (Solution A, $\mathrm{NaCl}, \mathrm{KH}_{2} \mathrm{PO}_{4}, \mathrm{C}_{6} \mathrm{H}_{6} \mathrm{O}_{6}, \mathrm{KCl}, \mathrm{NaHCO}_{3}$; Solution B, $\mathrm{Ca}^{2+}$ carrier A23187, DMSO, Solution C, DMSO; 
A

a

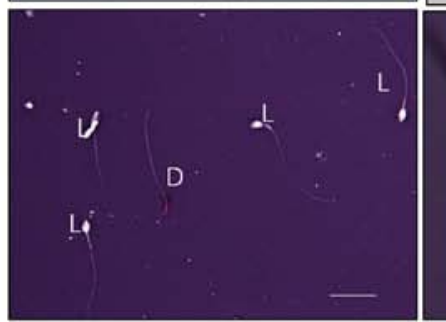

Fresh

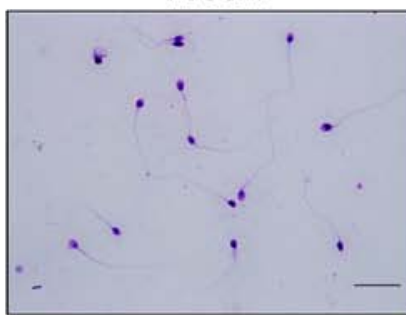

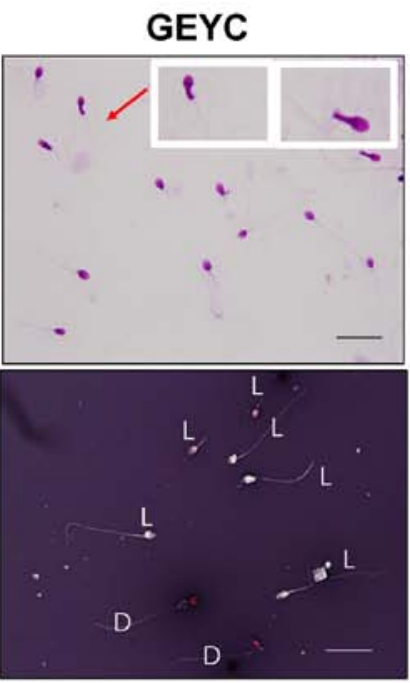

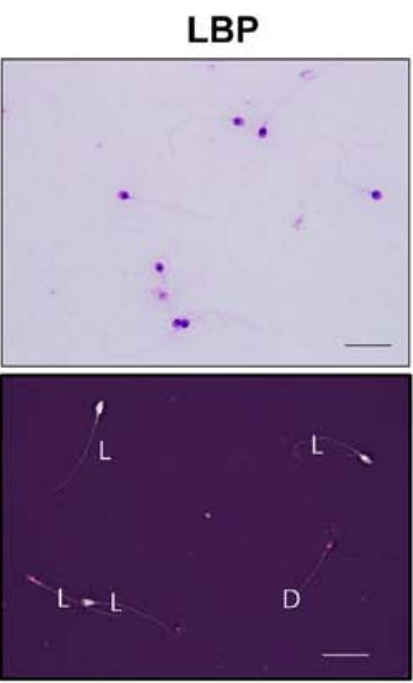

B

a

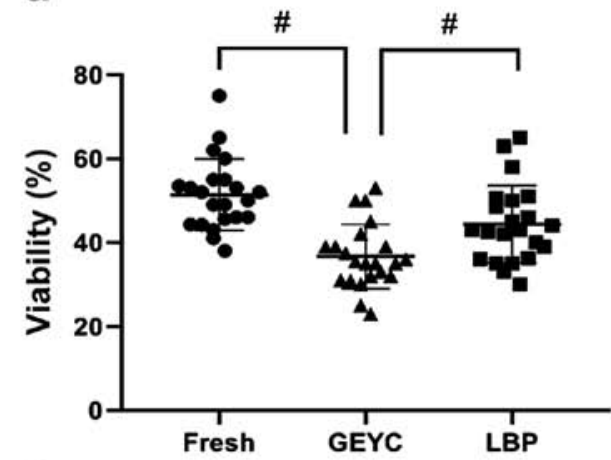

C

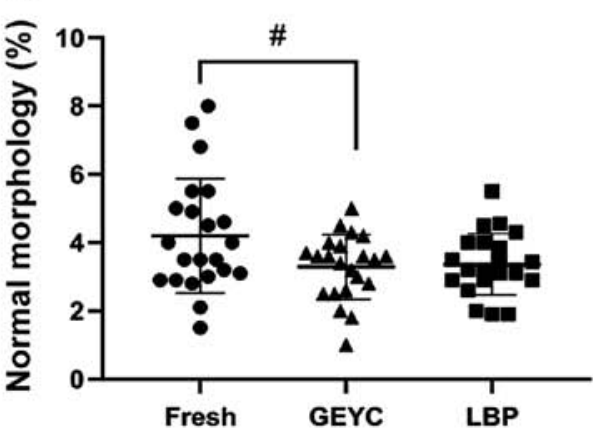

b

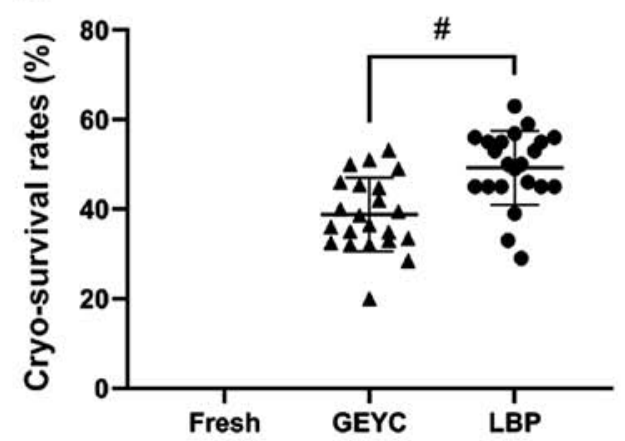

d

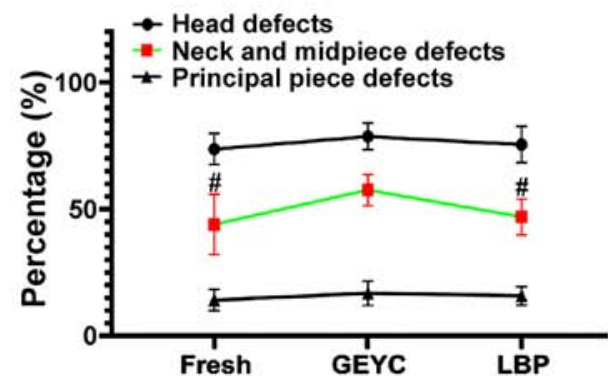

Figure 1. Analysis of the parameters of frozen sperm. (A) Microscopy images. (a) The first row presents diff Quik sperm staining (The white box marks two sperm with obvious abnormalities in the neck and the red arrow is used as the marker); (b) The second row displays eosin aniline black smear under the light field microscope (scale bar, $20 \mu \mathrm{m}$ ). (B) Quantified parameters of the sperm. (a) Sperm survival rate in each group; (b) sperm recovery rate after freezing; (c) sperm morphology analysis; (d) sperm abnormal morphology classification analysis. ( $\mathrm{n}=22$ ). "P<0.05 vs. GEYC group. GEYC, glycerin-yolk-citrate cryopreservative; LBP, Lycium barbarum polysaccharides; D, dead sperms were indicated by red or dark pink staining of the head; L, live sperms were indicated a white or light pink head.

Solution D, $\mathrm{NaCl}, \mathrm{KH}_{2} \mathrm{PO}_{4}, \mathrm{KCl}, \mathrm{NaH}_{2} \mathrm{PO}_{4}$; Solution E, Isothiocyanate-coupled pea agglutinin).

Detection of malondialdehyde (MDA). MDA was detected using thiobarbituric acid (TBA) colorimetry. The MDA test kit of Nanjing Jiancheng Biological Preparation Co., Ltd. was selected for testing. The operation was performed according to the manufacturer's protocol and the absorbance value at $532 \mathrm{~nm}$ wavelength. Was read using a spectrophotometer (1410101 Thermo Scientific ${ }^{\text {TM }}$ Multiskan ${ }^{\text {TM }}$ FC; Thermo Fisher Scientific, Inc.).
Detection of ROS. The sperm cell oxidative stress activity superoxide anion flow cytometry (cat. no. GMS10096; GenMed, Ltd.) test kit was used for the detection of ROS, and the operation was strictly in accordance with the manufacturer's protocol. In this assay, 2',7'-dichlorofluorescein diacetate is a completely free dye that passes through the cell membrane. Once it is oxidized by molecules including hydrogen peroxide, peroxides and peroxynitrite anions, it produces fluorescence (26). Using this assay, the concentration of ROS in spermatozoa were measured. The specific steps were as follows: Fresh semen $(1 \mathrm{ml})$ was added to a $1.5-\mathrm{ml}$ 
centrifuge tube, followed by centrifugation at room temperature for $10 \mathrm{~min}$ at a speed of $300 \mathrm{x} \mathrm{g}$. After discarding the supernatant, $500 \mathrm{ml}$ of reagent $\mathrm{C}$ was added, followed by thorough mixing. Subsequently, $25 \mu \mathrm{l}$ of dye A and $25 \mu \mathrm{l}$ of dye B was added to the aforementioned sperm cells. Following thorough mixing, the cells were incubated at room temperature $\left(25^{\circ} \mathrm{C}\right)$ for $40 \mathrm{~min}$ under the exclusion of light. The cells were then centrifuged at room temperature for $5 \mathrm{~min}$ at a speed of $300 \mathrm{x}$ g. The supernatant was carefully removed and $500 \mu \mathrm{l}$ of storage solution (reagent $\mathrm{C}$ ) was added. Following mixing, dual-channel analysis was immediately performed using the BD Accuri C6 (Beckton, Dickinson and Company) with an excitation wavelength at $488 \mathrm{~nm}$. The voltage of the FL1 and FL2 channels was regulated to make the major group appear in the specified gate. After the experiment, the data was imported into FlowJo version 10 (FlowJo LLC) for analysis. The sperm flow rate was controlled at $100 / \mathrm{sec}$, and at least 10,000 sperms were collected, and each sample was analyzed at least twice.

Immunofluorescence. The sperm smears were fixed in $4 \%$ paraformaldehyde at room temperature for $20 \mathrm{~min}$ and then washed with PBS three times, for $5 \mathrm{~min} /$ wash. The slides were placed in a humidified chamber and $0.30 \%$ Triton-X 100 (Beijing Solarbio Science \& Technology Co., Ltd.; T8200) was used to penetrate the membrane at room temperature for $10 \mathrm{~min}$. PBS was used to wash the slides thrice, for $5 \mathrm{~min} /$ wash. Subsequently, two drops of $3 \%$ peroxidase inhibitor were added to each slide to block the endogenous peroxidase at room temperature. The slides were incubated at room temperature for $10 \mathrm{~min}$ and then washed by PBS three times, for $5 \mathrm{~min} /$ wash. To block endogenous biotin and avidin, normal goat serum (Beijing Solarbio Science \& Technology Co., Ltd.; cat. no. SL038) was added to the sample, followed by incubation at room temperature for $10 \mathrm{~min}$. The samples were then incubated with primary antibodies in blocking solution overnight at $4^{\circ} \mathrm{C}$. The primary antibodies used were as follows: Rabbit anti-human Bcl-2 (cat. no., 12789-1-AP; ProteinTech Group, Inc.), Bax (cat. no. 50599-2-Ig; ProteinTech Group, Inc.), CytC (cat. no. 12245-1-AP; ProteinTech Group, Inc.), and caspase-3 (cat. no. 19677-1-AP; ProteinTech Group, Inc.) antibody (1:200 dilution; $50 \mu \mathrm{l} /$ tablet. BS was used instead of the primary antibody in the negative control group.

The slides were then incubated with secondary antibody (goat anti-rabbit IgG-H\&L (cat. no. ab6721; 1:1,000; Abcam) for $1 \mathrm{~h}$ at room temperature and after washing with PBS, samples were mounted with DAPI (cat. no. ZLI-9557; OriGene Technologies, Inc.). Confocal scanning images were captured using a DM 2000 microscope (x1,000; Leica Microsystems) with a digital camera (cat. no. DFC450C; Leica Microsystems).

Western blot analysis. Protein was extracted with a whole protein extraction kit (cat. no. KGP2100; Nanjing KeyGen Biotech Co., Ltd.) and protein was then quantified using the BCA method (cat. no. E162-01; Nanjing KeyGen Biotech Co., Ltd.). In each lane, 18-20 $\mu$ g protein lysate was separated by $10 \%$ SDS-PAGE and proteins were transferred to PVDF membranes (Merck KGaA) and the membranes were blocked in 5\% non-fat milk (blocking solution) at room temperature for $1 \mathrm{~h}$. The membrane was then incubated with primary antibodies overnight at $4^{\circ} \mathrm{C}$. The membranes were washed with Tris-buffered saline containing Tween-20 three times and then incubated with horseradish peroxidase-conjugated goat anti-rabbit IgG-H\&L (cat. no. ab6721; 1:5,000; Abcam) secondary antibody at room temperature for $1 \mathrm{~h}$. The PVDF membranes were then visualized using an enhanced chemiluminescence (ECL) detection system reagents and images were captured with the ChemiDocXRS (Bio-Rad Laboratories, Inc.). After the experiment, the images were assessed using ImageJ version 1.8.0 (National Institutes of Health). The primary antibodies were as follows: Caspase-3 (1:10,000; cat. no. 19677-1-AP; ProteinTech Group, Inc.), Bax (1:5,000; cat. no. 50599-2-Ig; ProteinTech Group, Inc.), Bcl-2 (1:10,000; cat. no., 12789-1-AP; ProteinTech Group, Inc.), CytC (1:1,000; cat. no. 12245-1-AP; ProteinTech Group, Inc.), GAPDH (1:10,000; cat. no. 10494-1-AP; ProteinTech Group, Inc.).

Determination of RNA levels. Total RNA from each group was extracted with a total RNA preparation kit (cat. no. AP-MN-MS-RNA-520G; Corning, Inc.) and reverse transcribed $\left(42^{\circ} \mathrm{C}\right.$ for $15 \mathrm{~min}$, then $85^{\circ} \mathrm{C}$ for $\left.5 \mathrm{sec}\right)$ into complementary (c)DNA using transcript all in one first-strand cDNA synthesis super mix (cat. no. AT341-02; TransGen Biotech Co., Ltd.). The SYBR premix ex taq II dye fluorescence quantitative kit (cat. no. RR820A; Takara Biotechnology Co., Ltd.) was used for PCR amplification in an ABI 7500 fast quantitative PCR instrument (Thermo Fisher Scientific, Inc.). After $30 \mathrm{sec}$ of denaturation at $95^{\circ} \mathrm{C}, 40$ cycles of amplification were performed: $5 \mathrm{sec}$ of denaturation at $95^{\circ} \mathrm{C}$, annealing/elongation at $60^{\circ} \mathrm{C}$ for $30 \mathrm{sec}$. The primer sequences were as follows: Caspase-3 forward, 5'-TGGAATGTCAGC TCGCAATG-3' and reverse, 5'-CAGGTCCGTTCGTTCCAA AA-3'; Bax forward, 5'-AGATCATGAAGACAGGGGCC-3' and reverse, 5'-ATCCTCTGCAGCTCCATGTT-3'; Bcl-2 forward, 5'-AACTCTTCAGGGATGGGGTG-3' and reverse, 5'-GCTGGGGCCATATAGTTCCA-3'; CytC forward, 5'GCCTCAATGTCCCTGCTTCT-3' and reverse, 5'-TCG TTTCCAGCATGAGTGCT-3'; GAPDH forward, 5'-AGTCTA CTGGCGTCTTCACC-3' and reverse, 5'-CCACGATGCCAA AGTTGTCA3'.

Transmission electron microscopy (TEM). After liquefaction, the semen samples were washed twice with $0.85 \% \mathrm{NaCl}$ and centrifuged at room temperature $1,000 \times \mathrm{g}$ for $10 \mathrm{~min}$. The supernatant was then discarded and then $2.5 \%$ glutaraldehyde was added slowly, and samples were fixed in a $4^{\circ} \mathrm{C}$ refrigerator for 2-4 h. Subsequently, the samples were fixed with osmium acid (1\%), washed with distilled water, embedded for $2 \mathrm{~h}$ at room temperature in Epon812 epoxy resin, cut into semi thin section $(1 \mathrm{~nm})$, stained at room temperature for $1 \mathrm{~h}$ by toluidine blue and positioned under a light microscope. The sperm cell area was subjected to semi thin section $(1 \mathrm{~nm})$ and a JEM1200-EX TEM (JEOL, Ltd.) was used to observe the changes of mitochondria in the sperm tail. An experienced electron microscopist blinded to the origin of the samples tested and recorded the information. The specific evaluation methods were as follows: Under an electron microscope, at least 100 sperm from each group of samples was evaluated, focusing on evaluation of the microstructure of mitochondria in the tail of the sperm, observing whether the arrangement of mitochondria was orderly, whether there was oedema and 
Table I. Analysis and detection of sperm motility related indexes by CASA.

\begin{tabular}{llccccc}
\hline Group & PR $(\%)$ & VCL $(\mu \mathrm{m} / \mathrm{sec})$ & VSL $(\mu \mathrm{m} / \mathrm{sec})$ & VAP $(\mu \mathrm{m} / \mathrm{sec})$ & ALH $(\mu \mathrm{m})$ & BCF $($ times $/ \mathrm{sec})$ \\
\hline Fresh & $22.0 \pm 5.2^{\mathrm{a}}$ & $38.5 \pm 3.5^{\mathrm{a}}$ & $19.7 \pm 3.8^{\mathrm{a}}$ & $22.7 \pm 2.2^{\mathrm{a}}$ & $4.4 \pm 1.1^{\mathrm{a}}$ & $13.3 \pm 1.5^{\mathrm{a}}$ \\
GEYC & $12.3 \pm 3.1$ & $26.5 \pm 3.1$ & $11.2 \pm 3.2$ & $13.0 \pm 2.6$ & $2.1 \pm 0.4$ & $9.3 \pm 1.4$ \\
LBP & $19.3 \pm 4.7^{\mathrm{a}}$ & $32.9 \pm 2.8^{\mathrm{a}, \mathrm{b}}$ & $16.5 \pm 3.4^{\mathrm{a}}$ & $20.9 \pm 3.4^{\mathrm{a}}$ & $2.4 \pm 0.5^{\mathrm{b}}$ & $10.2 \pm 1.1^{\mathrm{a}}$ \\
\hline
\end{tabular}

${ }^{\mathrm{a}} \mathrm{P}<0.05$ vs. GEYC group; ${ }^{\mathrm{b}} \mathrm{P}<0.05$ vs. Fresh group; $(\mathrm{n}=22)$. CASA was used to analyse sperm viability before and after cryopreservation. CASA, Computer-aided sperm analysis; PR, percentage of sperm forward movement; VCL, curvilinear velocity; VSL, sperm linear rate; VAP, sperm average path rate; ALH, sperm head swing range; BCF, sperm whip frequency; GEYC, glycerol-egg-yolk-citrate cryopreservative; LBP, Lycium barbarum polysaccharides.

the severity of oedema in mitochondria and whether there was damage to the structure of the mitochondrial membrane.

Statistical analysis. Values are expressed as the mean \pm standard error of the mean unless otherwise noted in the figure legends. Statistical analysis was performed using a SPSS 24.0 statistical package (IBM Corp.), differences among multiple groups were analyzed by Welch's one-way ANOVA, following by Tukey's HSD test. $\mathrm{P}<0.05$ was considered to indicate a statistically significant difference.

\section{Results}

The experimental images of sperm morphology and the sperm survival rate are presented in Fig. 1A, from which it can be seen that sperm neck abnormality (Fig. 1A-a) and sperm mortality (Fig. 1A-b) are indicated to increase during the freezing process. The results also indicated that the sperm viability decreased after sperm cryopreservation. The viability of the LBP group was higher compared with the GEYC group $(\mathrm{P}<0.05)$, however, the viability of the GEYC group was lower compared with the Fresh group $(\mathrm{P}<0.05)$, as presented in Fig. 1B-a. The sperm cryo-survival rate in the LBP group was also higher compared with the GEYC group $(\mathrm{P}<0.05)$, as indicated in Fig. 1B-b. The normal morphology rates of GEYC group was lower compared with the Fresh group $(\mathrm{P}<0.05)$, and there was no obvious difference between the LBP group and the GEYC group (Fig. 1B-c), but the abnormal morphology rate at the middle part of sperms in the LBP group was significantly lower compared with the GEYC group $(\mathrm{P}<0.05)$, the abnormal morphology rate at the middle part of sperms in the GEYC group was significantly higher compared with the Fresh group $(\mathrm{P}<0.05)$ as presented in Fig. 1B-d. After cryo-survival, the sperms' progressive motility, VCL, VSL and VAP, BCF in the LBP group were higher compared with those in the GEYC group $(\mathrm{P}<0.05)$, the sperm progressive motility, VCL, VSL, VAP, BCF and ALH in the GEYC group were lower compared with those in the Fresh group $(\mathrm{P}<0.05)$ as presented in Table I.

Sperm function. To assess the DFI, a minimum of 5,000 cells from each sample were acquired and analyzed using fluorescence-assisted cell sorting interfaced with a data analysis software. Sperm DFI was assessed using a SCSA. The green fluorescence was that of acridine orange dye binding to double-stranded DNA, while the red fluorescence was that of acridine orange dye binding to single-stranded DNA. As presented in Fig. 2A, following sperm cryopreservation, the DFI rose and the DFI of the LBP group $(27.3 \pm 5.1 \%)$ was lower compared with the GEYC group $(38.2 \pm 5.6 \%)$ but significantly higher compared with the Fresh group (17.5 $\pm 3.1 \%)$.

JC-1 dye molecules accumulate in the mitochondrial matrix and produce red fluorescence; however, if the MMP is low, the JC-1 dye is not able to accumulate in the mitochondrial matrix and green fluorescence is then produced. As presented in Fig. 2B, the MMP of cryopreserved spermatozoa was increased. The MMP of the LBP group (39.2 $\pm 6.1 \%)$ was higher compared with the GEYC group $(28.8 \pm 7.0 \%)$ but significantly lower compared with the Fresh group $(49.8 \pm 9.5 \%)$.

After the acrosome reaction was induced, the relative fluorescence intensity was weaker compared with the control, indicating that the fluorescence peak shifted to the left. The AR of cryopreserved sperm was reduced and there was no significant difference between the LBP group (17.7 $\pm 3.3 \%)$ and the GEYC group (14.7 $\pm 2.5 \%)$; The AR of the GEYC group was

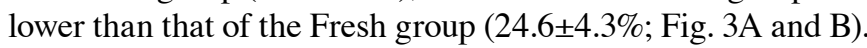

Apoptosis-associated proteins. The results of the immunofluorescence analysis indicated that the expression of Bcl-2, Bax, CytC and caspase- 3 was concentrated in the head and middle part of the sperms (Fig. 4A). Western blot analysis suggested that the LBP group had a higher content of Bcl-2 protein compared with the GEYC group, while the expression levels of Bax, CytC and caspase-3 protein were lower compared with the GEYC group. Bcl-2, CytC, Caspase-3 in the LBP group and Fresh group differed significantly, while the differences in Bax were not significant, as presented in Fig. 4C. The results of the reverse transcription PCR analysis indicated that, compared with the GEYC group, Bax, CytC and caspase-3 were significantly decreased in the LBP group. Bcl-2 in the LBP group was higher compared with the GEYC group, Bcl-2, Bax, CytC, Caspase-3 in the LBP group and Fresh group differed significantly, as presented in Fig. 4D.

TEM and ROS detection results. TEM was used to detect the microstructure of sperm. It was indicated that the mitochondrial structure in the middle part of the sperm tail was severely damaged after freezing, the mitochondrial cristae structure was disorganized and normal mitochondrial cristae disappeared. By contrast, there was a significant improvement in the LBP group. Specifically, the mitochondria at the sperm neck 
A
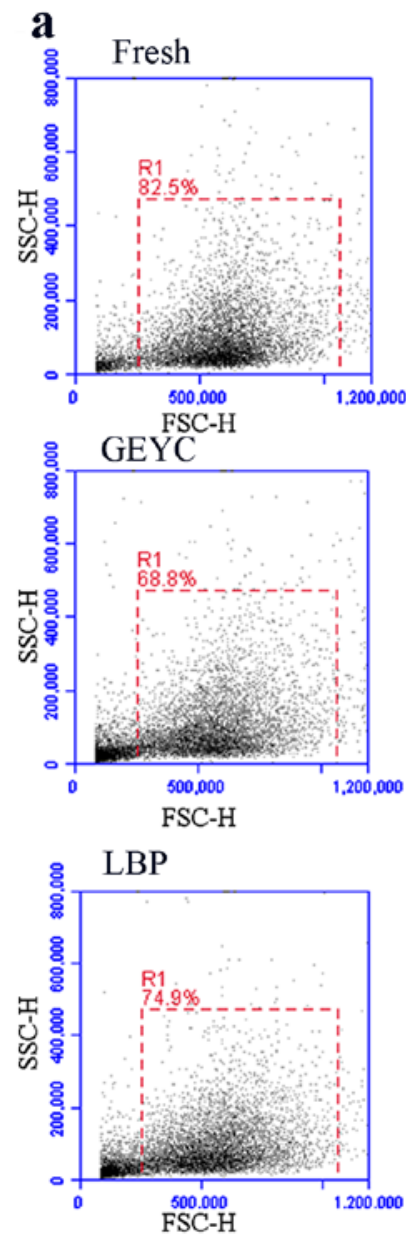

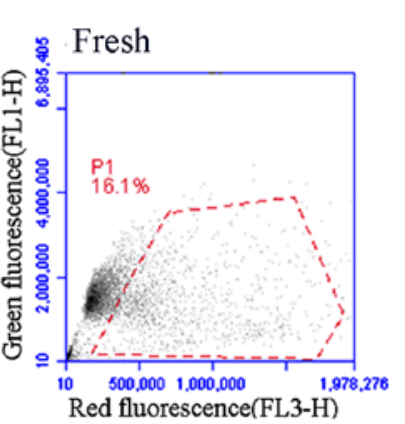

GEYC
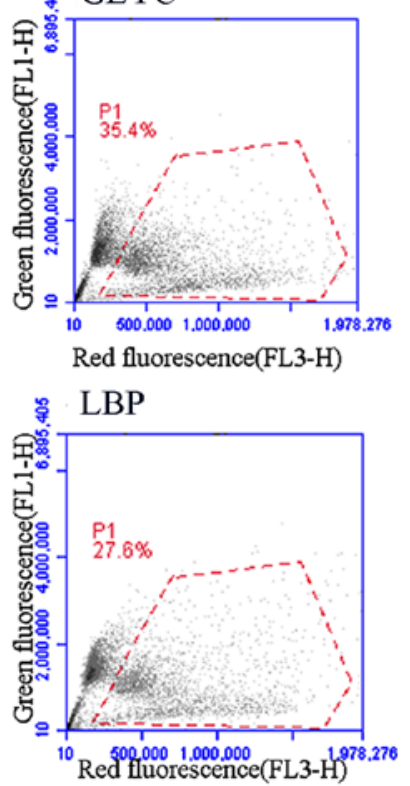

B

a
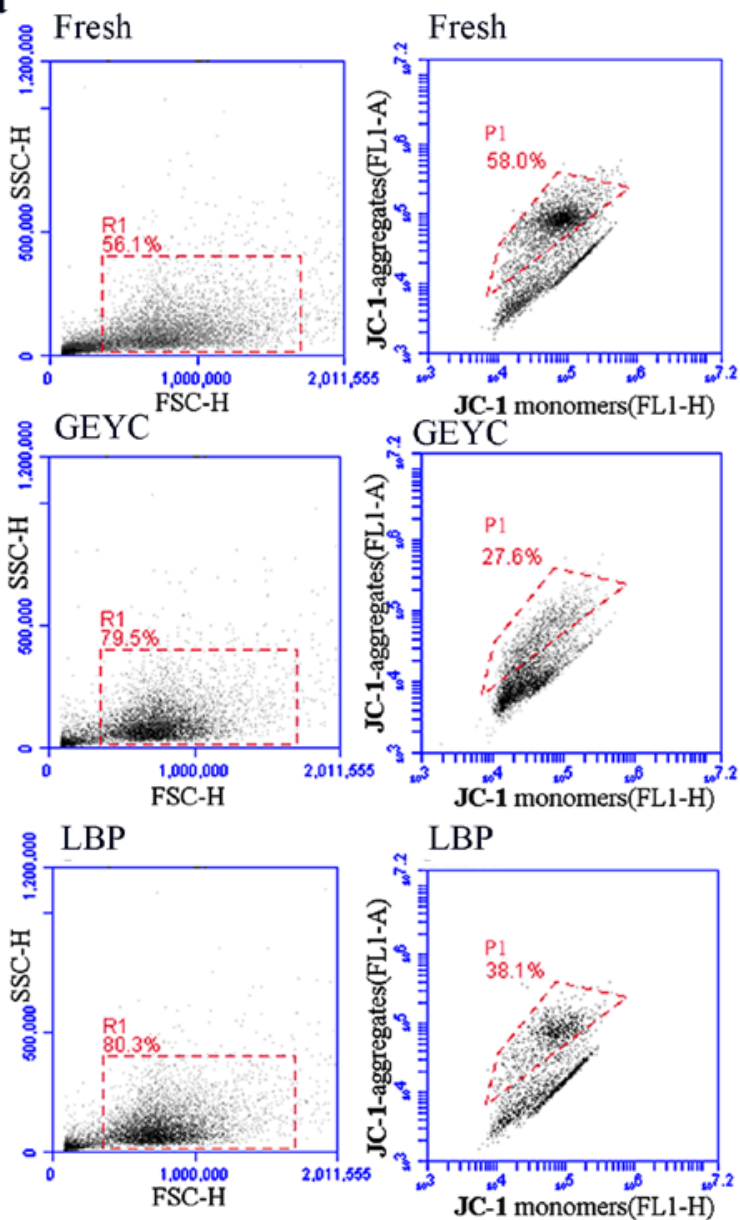

\section{A-b}

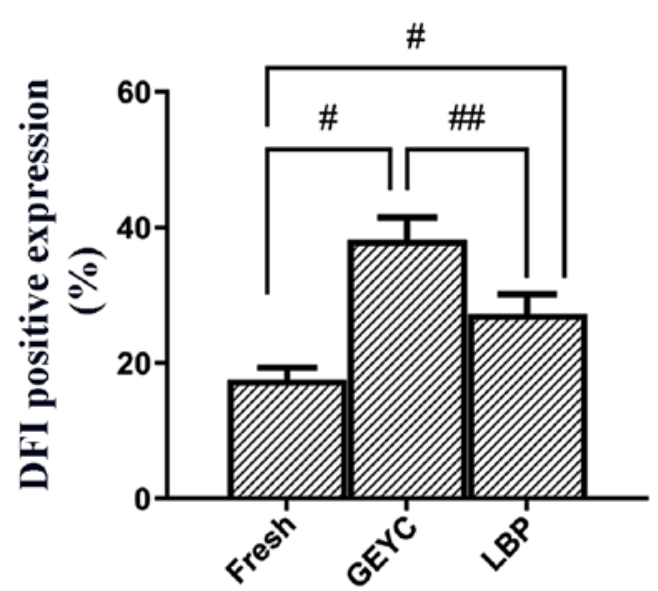

B-b

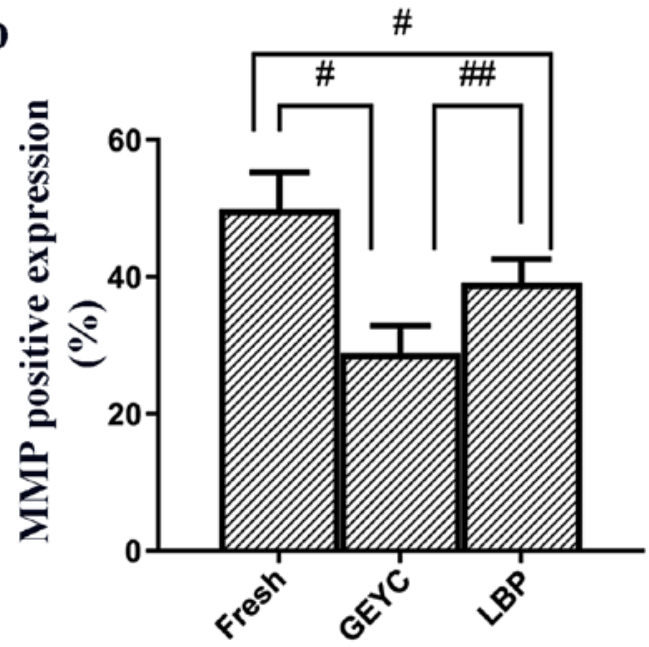

Figure 2. (A-a) FSC/SSC dot plot (52) (R1) is indicated in the left-hand panel and the cytogram (red vs. green fluorescence) in the right-hand panel, SSC-H reflects the fluorescence concentration per unit area of cells. (A-b) Quantitative analysis of the fluorescence-assisted cell sorting data (DFI). (B-a) The scattergate and point diagram of representative sperm samples stained by JC-1. In the right-hand panel, P1 contains high-mitochondrial membrane potential sperm subsets. (B-b) Quantitative analysis of the fluorescence-assisted cell sorting data (MMP). $(\mathrm{n}=22) .{ }^{\#} \mathrm{P}<0.05$ vs. Fresh group; ${ }^{\# \#} \mathrm{P}<0.05$ vs. GEYC group. GEYC, glycerin-yolk-citrate cryopreservative; LBP, Lycium barbarum polysaccharides; FSC-H, forward scatter-height; SSC, side scatter; MMP, mitochondrial membrane potential; AR, acrosome reaction; DFI, DNA fragmentation index; R1, region 1; FL, fluorescence.

were orderly arranged and abnormal mitochondrial structures were significantly less frequent, as presented in Fig. 5A-B.

The TBA colorimetric method was used for MDA detection. The results suggested that the LBP group $(18.0 \pm 3.6 \mathrm{nmol} / \mathrm{ml})$ had a significantly lower sperm MDA content than the GEYC group $(23.9 \pm 4.1 \mathrm{nmol} / \mathrm{ml})$, which was higher than the fresh group $(16.3 \pm 3.4 \mathrm{nmol} / \mathrm{ml})$, but there was no significant difference between the LBP group and the Fresh group. ROS 

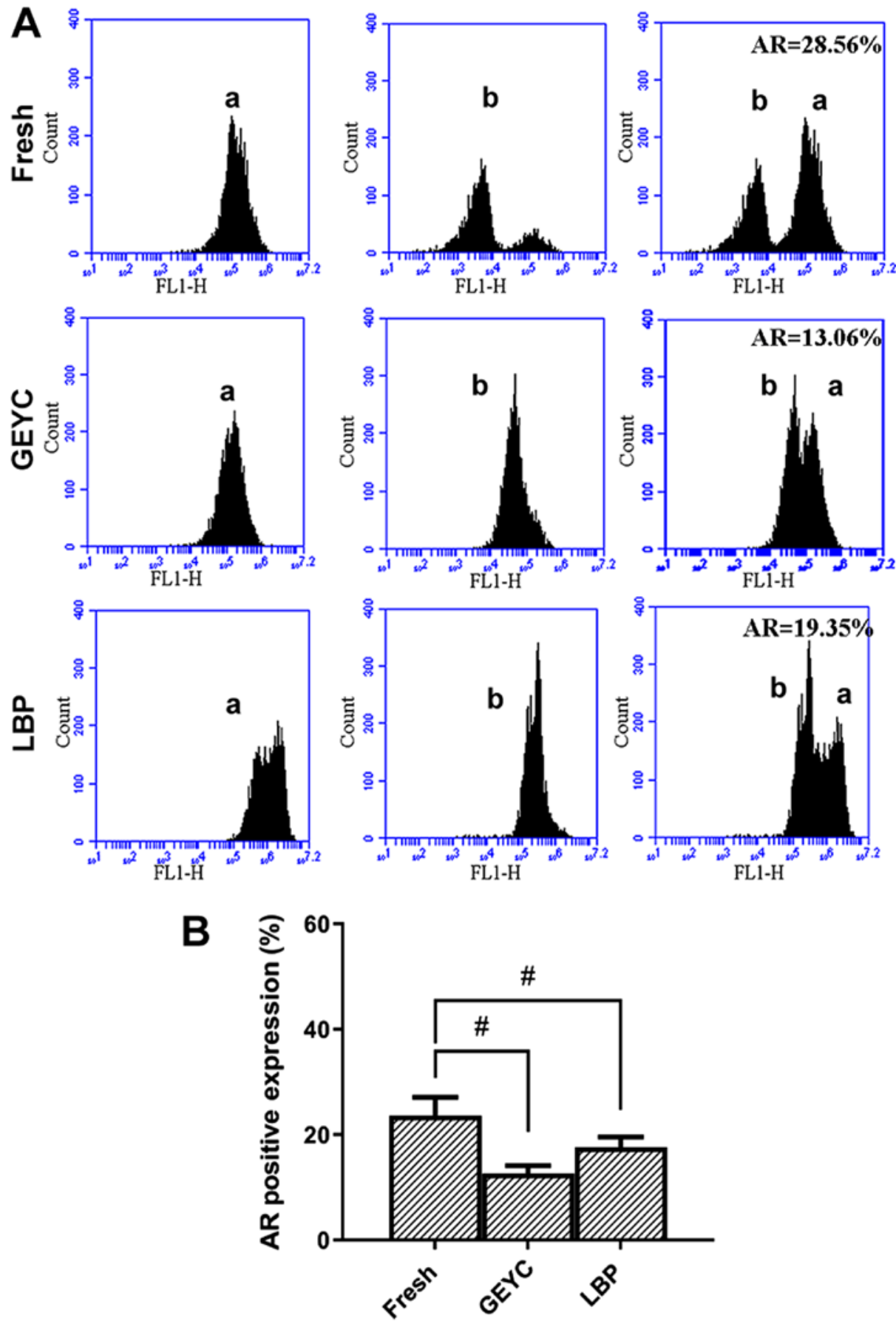

Figure 3. (A) Histograms displaying the distribution of acrosome fluorescence in sperm in (a) the absence and (b) presence of the calcium ionophore A23187. (B) Quantitative analysis of the fluorescence-assisted cell sorting data. ( $\mathrm{n}=22) .{ }^{\#} \mathrm{P}<0.05$ vs. Fresh group. GEYC, glycerol-egg-yolk-citrate cryopreservative; LBP, Lycium barbarum polysaccharides; AR, acrosome reaction.

detection based on flow cytometry indicated that the rate of ROS-positive cells in the LBP group (38.1 $\pm 6.7 \%)$ was significantly lower compared with the GEYC group $(58.3 \pm 10.8 \%)$, and this rate in the LBP group increased as compared with the Fresh group (17.4 $\pm 5.2 \%$; $\mathrm{P}<0.05$; Table II; Fig. 5C).

\section{Discussion}

With the development of assisted reproductive technology, the cryopreservation of germ cells is improving. The selection of protective agents is progressing, and the cryopreservation scheme is undergoing constant optimization and innovation. The formation of ice crystals during freezing-thawing, the production of massive ROS and the toxic effect of glycerol in GEYC cryopreservative on cells all seriously affect the quality and mobility of sperm after freezing (27-29). Specifically, the ROS generated during the freeze-thawing process may damage the mitochondrial membrane $(30,31)$ and reduce the MMP of sperm mitochondria (32), thereby leading to a sharp decline in sperm motility. Sperm plasma membranes are vulnerable to oxidative stress, as they are rich in polyunsaturated fatty acids $(33,34)$. Furthermore, oxidative damage may lead to sperm plasma 

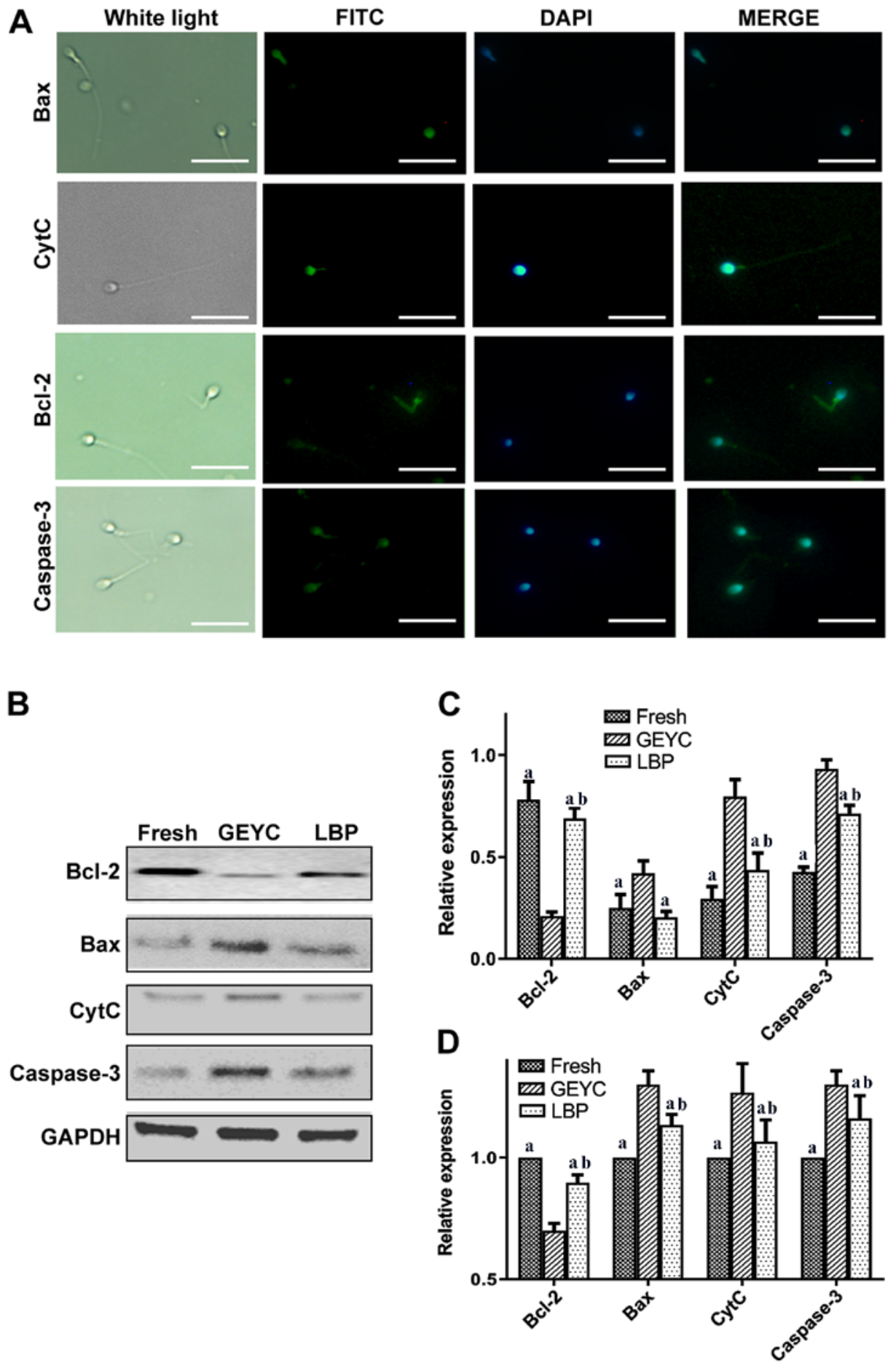

Figure 4. Effect of LBP on the expression of genes of the mitochondrial pathway of apoptosis, Bcl-2, Bax, CytC and caspase-3. (A) Sperm immunofluorescence. The nuclei were counterstained with DAPI. (scale bar, $20 \mu \mathrm{m}$ ). (B) Western blot analysis of the protein expression of Bcl-2, Bax, CytC and caspase-3. (C) Quantitative grey value analysis of the protein expression levels from B. (D) mRNA expression levels detected by reverse transcription-quantitative PCR. $(\mathrm{n}=22) .{ }^{\mathrm{a}} \mathrm{P}<0.05$ vs. GEYC group; ${ }^{\mathrm{b}} \mathrm{P}<0.05$ vs. fresh group. GEYC, glycerol-egg-yolk-citrate cryopreservative; LBP, Lycium barbarum polysaccharides; CytC, cytochrome C.

membrane injury, respiratory depression, intracellular enzyme loss, axoneme protein damage and mitochondrial membrane injury $(1,9)$. In addition, the structure of sperm is damaged after freezing and thawing, the integrity of the sperm plasma membrane is broken, the sperm tail is injured, the mitochondrial structure and function are impaired, ATP malfunctions occur, the sperm motility parameters decrease significantly and sperm function is damaged. Due to this, sperm are weakened and their survival impaired after cryo-preservation, having a negative impact on its fertilization ability (35-40).

The damage to sperm that is caused by excessive ROS produced by freezing and thawing is reduced by the addition of antioxidants, and thus, sperm motility may be improved. For instance, Zheng et al (41) reported that the addition of a certain concentration of $\mathrm{Vit} E$ to the sperm cryopreservative may reduce sperm damage that is caused by the freeze-thawing process 

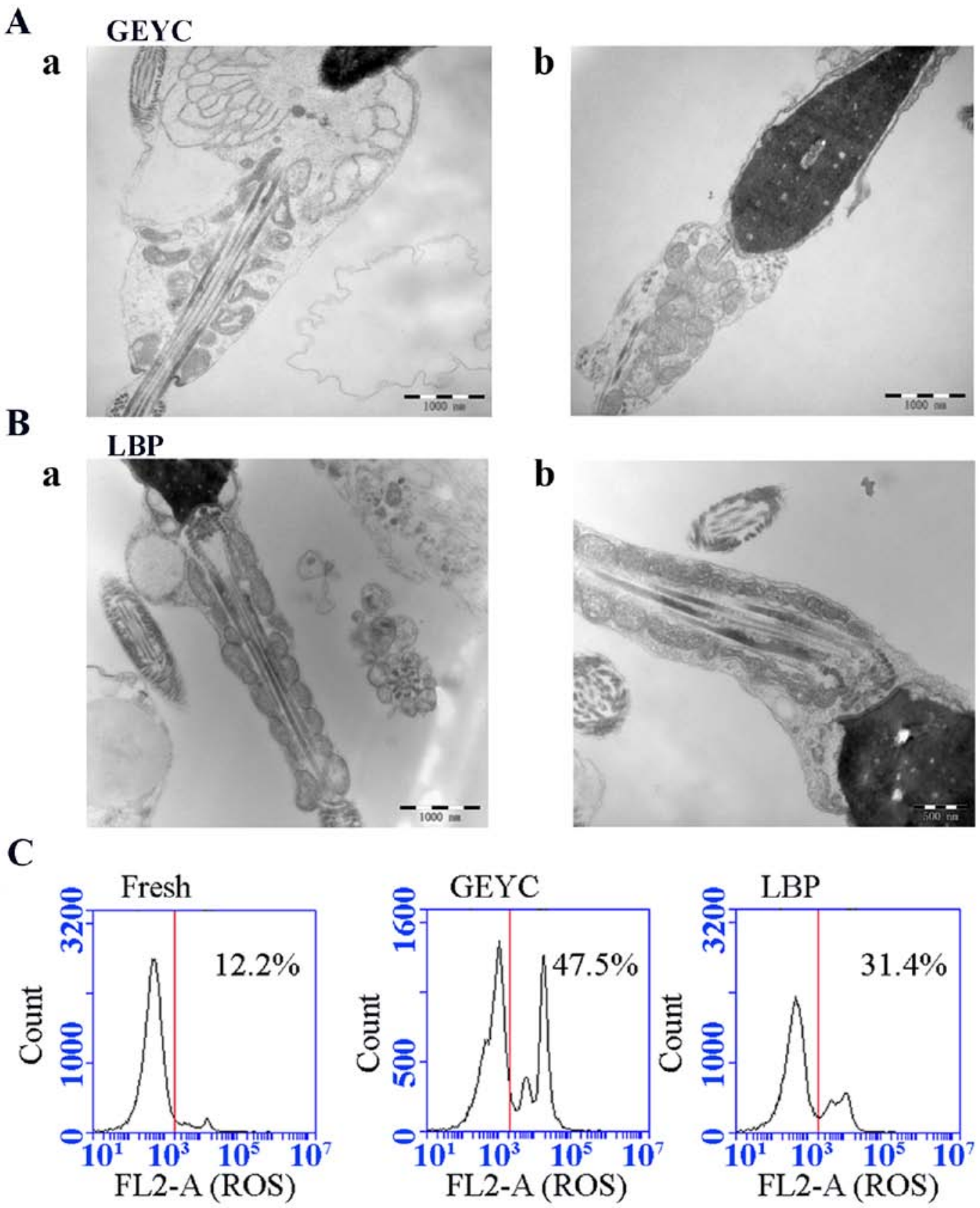

Figure 5. Detection of the mitochondrial microstructure and ROS in sperm. Sperm microstructure in (A) Sperm microstructure in the glycerin-yolk-citrate cryopreservative group: (A-a)The plasma membrane and mitochondrial membrane of sperm are swollen and damaged. (A-b) Sperm mitochondria are disordered and piled up. (B) Sperm microstructure in the Lycium barbarum polysaccharides group: (B-a) Themitochondria of sperm arranged in order. (B-b) Mitochondrial cristae can be seen in sperm mitochondria. (scale bar, 1,000 nm). (C) ROS fluorescence histogram of each group. ROS increased and the peak shifted to the right. ROS, reactive oxygen species; GEYC, glycerol-egg-yolk-citrate cryopreservative; LBP, Lycium barbarum polysaccharides.

and partly improve the motility, survival rate and DNA integrity of sperm. However, most previous studies are concentrated on sperm viability and morphology after freezing, rather than the impact on sperm microstructure $(7-9,41)$. There are few studies eluding to the role of antioxidants in sperm freezing and thawing and indicating which pathways and molecules are affected during this process $(10-13,35)$.

LBP, which is extracted from a traditional Chinese medicinal plant named Lycium barbarum, exhibits biological effects including antioxidative effects, scavenging of oxygen free radicals and the stabilization of the cell membrane (15). In a previous study, LBP was added at different concentrations to conventional GEYC cryopreservative and it was determined that LBP at $1,000 \mu \mathrm{g} / \mathrm{ml}$ had the most significant effect in improving sperm motility after freezing, and for this reason, the concentration of the added LBP in the present study was $1,000 \mu \mathrm{g} / \mathrm{ml}$ (Fig. 6). The results indicated although there was no significant difference between the LBP group and the GEYC group in terms of the normal sperm morphology rate, the deformity rate of the sperm tail at the middle part in the LBP group was significantly declined as compared with that the GEYC group. TEM observation indicated that 
Table II. MDA and ROS in sperm.

\begin{tabular}{lcc}
\hline Group & MDA $(\mathrm{nmol} / \mathrm{ml})$ & ROS $(\%)$ \\
\hline Fresh & $16.3 \pm 3.4^{\mathrm{a}}$ & $17.4 \pm 5.2^{\mathrm{a}}$ \\
GEYC & $23.9 \pm 4.1$ & $58.3 \pm 10.8$ \\
LBP & $18.0 \pm 3.6^{\mathrm{a}}$ & $38.1 \pm 6.7^{\mathrm{a}, \mathrm{b}}$ \\
\hline
\end{tabular}

${ }^{\mathrm{a}} \mathrm{P}<0.05$ vs. GEYC group; ${ }^{\mathrm{b}} \mathrm{P}<0.05$ vs. Fresh group; ( $\left.\mathrm{n}=22\right)$. GEYC, glycerol-egg-yolk-citrate cryopreservative; LBP, Lycium barbarum polysaccharides; ROS, reactive oxygenspecies; MDA,malondialdehyde.

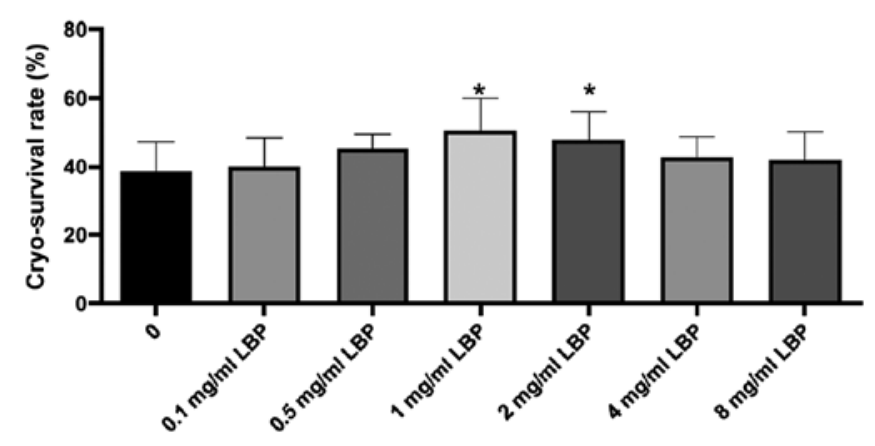

Figure 6. Effect of Lycium barbarum polysaccharide of different concentrations on sperm recovery rate. ${ }^{*} \mathrm{P}<0.05 \mathrm{vs} .0 \mathrm{mg} / \mathrm{ml}$ group.

the mitochondrial area in the middle part of the sperm tail was obviously swollen, the normal mitochondrial cristae disappeared, and the mitochondrial structure was disorganized. In the LBP group, the ultrastructures of mitochondria and abnormal spermatozoa in the neck were significantly improved.

The energy required for sperm motility is derived from the ATP produced by mitochondria and the structure and functional integrity of mitochondria after freezing is important for sperm motility and quality (42). The MMP in the frozen group with LBP was significantly higher compared with the GEYC group $(\mathrm{P}<0.05)$ and the DFI of sperm declined after LBP was added, which may be attributed to the biological effects of LBP to act as an antioxidant, scavenge oxygen free radicals and stabilize the cell membrane. In the present study, the AR detection result in the LBP group was higher compared with the GEYC group but the difference was not statistically significant, suggesting that LBP could promote sperm acrosome reaction, but LBP at this concentration did not have a significant promoting effect on every sample. This may be related to the difference of each sperm sample, such as the difference in the redox ability. If the sample size was expanded, the current study may have gained more satisfactory results. Furthermore, the addition of LBP reduced the contents of MDA and ROS, which demonstrated that LBP exhibits an antioxidant capacity. A large number of previous studies indicated that LBP may scavenge oxygen free radicals, increase SOD levels in cells and withstand oxidation (43-45). In the present study, after LBP was added to the cryopreservative, the sperm forward motility and movement parameters (VCL, VSL and VAP) increased significantly, and the MMP and DFI were also markedly improved, indicating that the antioxidant capacity of LBP reduced the damage to sperm DNA and mitochondrial membrane caused by ROS, thereby protecting mitochondria and DNA.

The mitochondrial signaling pathway is a common apoptosis pathway that may be activated by a number of different factors to trigger the interaction between $\mathrm{Bcl}-2$ and Bax, causing the latter to oligomerize and be inserted into the mitochondrial membrane, leading to changes in the permeability of the mitochondrial membrane and release of CytC. Subsequently, the caspase cascade is initiated and the downstream caspase-3 is activated, resulting in cell apoptosis (46). Hezavehei et al (47) indicated that freezing resulted in an increase of caspase-3 and apoptosis of human sperm. Therefore, four key genes, namely Bcl-2, Bax, CytC and caspase-3, were used as indicators to observe the effect of LBP on sperm freezing and thawing in the present study. The protein imprinting results indicated that in the LBP group, Bcl-2 increased compared with the GEYC group, while Bax, CytC and caspase- 3 decreased, indicating that LBP affected the key molecules in this pathway and inhibited cell apoptosis. The detection results for RNA levels were consistent with those for the protein levels. The biological functions of LBP to exert antioxidant effects and scavenge oxygen free radicals attenuate the effect of excessive ROS on the sperm mitochondrial pathway in the freeze-thawing process, thereby protecting the sperms' mitochondria and function. Similar to the results of the present study, Dai et al (48) reported that the addition of LBP when freezing goat spermatogonial stem cells led to the upregulation of the expression of anti-apoptotic $\mathrm{Bcl}-2$ and downregulation of Bax protein, which significantly reduced apoptosis of the cells. Ma et al (49) and Huang et al (50) indicated that LBP my reduce CytC and caspase-3 levels, thus significantly reducing apoptosis of the studied cells. In addition, Li et al (51) added LBP when freezing goat semen and revealed that the integrity rate of the sperm plasma membrane and the mitochondrial activity were significantly increased.

In the sperm treatment process of the present study, sperm was separated from semen using centrifugation after semen liquefaction and then cultured in BWW medium with LBP for 20 min prior to freezing, so that LBP was able to better interact with sperm and obtain a better freezing effect. In this way, the damage of non-sperm components to the structure and function of sperms in the semen, as well as any interference with the experimental results, were avoided.

Lycium barbarum is a traditional medicinal plant in China, and research into its major functional component, LBP, is still ongoing. Further study should be performed on the protective mechanism of LBP in sperm cryopreservation. With the continuous advance of various omics methods, it is possible to explore whether LBP has an interactive molecular target and further study its function, to develop a safer natural sperm cryopreservative and ultimately to improve the preservation of sperm.

\section{Acknowledgements}

Not applicable.

\section{Funding}

The current study was supported by the Natural Science Foundation of Ningxia (grant no. NZ17140), the Key R and 
D Plan Project of Ningxia Hui Autonomous Region (grant no. 2020BFH02002), Guangzhou Science and Technology Plan Project (grant no. 201707010394) and the open project of National Health Committee Key Laboratory of Male Reproduction and Genetics (grant no. KF201709).

\section{Availability of data and materials}

The datasets used and/or analyzed during the present study are available from the corresponding author on reasonable request.

\section{Authors' contributions}

HW and YT designed the study, BY and XZ performed sperm function tests, TEM and sperm protein and RNA analyses; SJ and YZ performed sperm motility tests and morphological tests. JT and JW performed the statistical analysis of the data in this study. HW and YT wrote and edited the paper. All authors read and approved the final manuscript.

\section{Ethics approval and consent to participate}

The present study was approved by the ethics committee of the General Hospital of Ningxia Medical University (approval no. 2019-402).

\section{Patient consent for publication}

Not applicable.

\section{Competing interests}

The authors declare that they have no competing interests.

\section{References}

1. Shabani Nashtaei M, Nekoonam S, Naji M, Bakhshalizadeh S and Amidi F: Cryoprotective effect of resveratrol on DNA damage and crucial human sperm messenger RNAs, possibly through 5' AMP-activated protein kinase activation. Cell Tissue Bank 19: 87-95, 2018

2. Li SJ, Su WD, Qiu LJ, et al: Protective effect of resveratrol on human sperm freezing injury. Chin J Androl 24: 499-503, 2018.

3. Nie ZY, Wu HF, Zhang N, et al: The effect of male age on sperm oxidative stress and nuclear DNA damage. Chin J Fam Planning 20: 30, 2012 (In Chinese).

4. Ma CJ, Zhuang JM, Deng SM, et al: The effect of docosahexaenoic acid added to glycerin yolk citrate cryoprotectors on the kinematical parameters of human sperm before and after cryopreservation. Chin Contemporary Med 25: 4-8, 2018 (In Chinese).

5. Chen ZW, Fan LQ, Wen RQ, Lu WH, et al: Assisted reproductive male technology. 1st edition. Beijing, People's Health Press, 2016.

6. Li Y: Study on the cryopreservation effect of Epimedium Polysaccharide on goat semen. Northwest Univ Agriculture Forestry Sci Technol, 2019.

7. Ren F, Feng T, Dai G, Wang Y, Zhu H and Hu J: Lycopene and alpha-lipoic acid improve semen antioxidant enzymes activity and cashmere goat sperm function after cryopreservation. Cryobiology 84: 27-3, 2018.

8. Losano JDA, Angrimani DSR, Rui BR, Bicudo LC, Dalmazzo A, Silva BCS, Viana CHC, Mendes CM, Assumpção MEOA, Barnabe VH and Nichi M: The addition of docosahexaenoic acid (DHA) and antioxidants (glutathione peroxidase and superoxide dismutase) in extenders to epididymal sperm cryopreservation in bulls. Zygote 26: 199-206, 2018.

9. Tian J, Zhang SH, Ma K, et al: The role of Rho/rock signal pathway in sperm anti freezing injury. Chin J Androl 4: 322-328, 2019 (In Chinese).
10. Shahrzad E, Zahiri S, Ghasemi F and Jahromi HK: A study of effects of L-carnitine on morphology and apoptosis in cryopreserved Sperm. Adv Environmental Biol 7: 2126-2134, 2013.

11. Yujie Z, Jing Y, Tailang Y, et al: The effect of acetyl-L-carnitine on oxidative damage of human sperm during cryopreservation. J Wuhan Univ (Med Ed) 35: 426-431, 2014.

12. Yujie Z, et al: The protective effect of acetyl-L-carnitine on acrosome integrity and ultrastructure of human sperm before and after cryopreservation. J Med Mol Biol 14: 268-273, 2017.

13. Li L, et al: Protective effect of mitoquinone, a mitochondrial targeted antioxidant, on oxidative stress injury of human sperm during freezing thawing. Chin J Androl 22: 205-211, 2016.

14. BerkovitzA,FitoussiD,ZhakovD andBreitbartH:Cryopreservation of human sperm in the presence of $\mathrm{Zn}^{2+}$ increases the motility rate. J Obstetrics Gynecological Investigations 1: 6-12, 2018.

15. Wu W, Xue SJ, Zhu LQ, et al: Study on the protective effect of Lycium barbarum polysaccharide on the injury of vascular endothelial cells induced by hydrogen peroxide through antioxidation and anti apoptosis. Shi Zhen Guo Yi Guo Yao 30: 1047-1049, 2019.

16. Rui Z, Kang KA, Mei JP, Kim KC, Kim AD, Chae S, Park JS, Youn UJ and Hyun JW: Cytoprotective effect of the fruits of Lycium Chinense Miller against oxidative stress-induced hepatotoxicity. J Ethnopharmacol 130: 299-306, 2010.

17. Yufang C, Yanrong W, Bin K, et al: Effect of Lycium barbarum polysaccharide on cryopreservation of fetal ovarian tissue. China Tissue Engineering Res 16: 7475-7479, 2012.

18. Lianghong M, Zhijun Q, Shengnan Y, et al: Effect of Lycium barbarum polysaccharide on proliferation of spermatogonial stem cells in vitro. China Tissue Engineering Res 15: 4277-4281, 2011.

19. Huan Z, Na T, Lihua W, Jianan P, Yi D and Menghua C: Lycium barbarum polysaccharides alleviate kidney injury and oxidative stress in unilateral ureteral obstruction rats. Chin J Nephrol 34: 377-384, 2018.

20. Vittoria MV, Pasciu V, Gadau SD, Baralla E, Serra E, Palomba D and Demontis MP: Possible antioxidant effect of Lycium barbarum polysaccharides on hepatic cadmium-induced oxidative stress in rats. Environmental Sci Pollution Res 24: 2946-2955, 2017.

21. Qingqing H, Bei Y, Tao Y, Fang M and Juan W: The protective effect of Lycium barbarum polysaccharide on the washing process of spermatozoa after freezing. Ningxia Med J 41: 1073-1075, 2019.

22. Zhang WD, Zhang Z, Jia LT, Zhang LL, Fu T, Li YS, Wang P, Sun L, Shi Y and Zhang HZ: Oxygen free radicals and mitochondrial signaling in oligospermia and asthenospermia. Mol Med Rep 10: 1875-1880, 2014.

23. World Health Organization, Department of Reproductive Health and Research: WHO laboratory manual for the Examination and processing of human semen, Fifth edition. Switzerland, WHO Press, 2010.

24. Lu JC, Jing J, Chen L, Ge YF, Feng RX, Liang YJ and Yao B: Analysis of human sperm DNA fragmentation index (DFI) related factors: A report of 1010 subfertile men in China. Reproductive Biol Endocrinol 16: 23, 2018.

25. Guowei Z, Zhi W, Xi L, Zou P, Yang H, Chen Q, Zhon N, Sun L, Gao J, Zhon Z, et al: Mitochondrial biomarkers reflect semen quality: Results from the MARCHS study in Chongqing, China. PLoS One 11: e0168823, 2016.

26. Zhao ZH, Zhang TT and Wang H: Effect of breviscapine on apoptosis of cortical neurons induced by hypoxia/reoxygenation. Practical Med Clin 1: 9-13, 2020.

27. Ma CJ, Jiang F,Zhuang JM, et al: The effect of glycerol maohuang sodium citrate cryoprotectors on human sperm motility parameters without cryopreservation. Chin J Androl 24: 37-39, 2010.

28. Zhao K, Chen W, Xu AM, et al: Preliminary study on the protective effect of isocitrate on sperm freezing and thawing. J Nanjing Med Univ Nat Sci Ed 37: 165-168, 2017.

29. Cheng S, Yan JQ, Zhu JQ, et al: DNA damage of Pseudosciaena crocea sperm cryopreserved with glycerol as antifreeze. China J Animal Husbandry 49: 34-36, 2013.

30. Dong QY, Shi H and Li JY: Research on cryopreservation injury and protection of human sperm. Chin J Fam Planning 27: 1259-1263, 2019.

31. Fu LL, Zhang LY, et al: The effect of levocarnitine on the motility parameters and mitochondrial function of frozen sperm. Chin J Androl 24: 1059-1063, 2018.

32. Gürler H, Molama E, Heppelmann M, Calisici O, Leiding C, Kastelic JP and Bollwein H: Effects of cryopreservation on sperm viability, synthesis of reactive oxygen species, and DNA damage of bovine sperm. Theriogenology 86: 562-571, 2016. 
33. Baumber J, Ball BA, Linfor JJ and Meyers SA: Reactive oxygen species and cryopreservation promote DNA fragmentation in equine spermatozoa. J Androl 24: 621-628, 2003.

34. Johnson AE, Freeman EW, Wildt DE and Songsasen N: Spermatozoa from the maned wolf (Chrysocyon brachyurus) display typical canid hyper-sensitivity to osmotic and freezing-induced injury, but respond favorably to dimethyl sulfoxide. Cryobiology 68: 361-370, 2014.

35. Zribi N, Chakroun NF, Ben Abdallah F, Elleuch H, Sellami A Gargouri J, Rebai T, Fakhfakh F and Keskes LA: Effect of freezing-thawing process and quercetin on human sperm survival and DNA integrity. Cryobiology 65: 326-331, 2012.

36. Chen SJ, Allam JP, Duan YG and Haidl G: Influence of reactive oxygen species on human sperm functions and fertilizing capacity including therapeutical approaches. Arch Gynecol Obstet 288: 191-199, 2013.

37. Ribas-Maynou J, García-Peiró A, Fernández-Encinas A, Abad C Amengual MJ, Prada E, Navarro J and Benet J: Comprehensive analysis of sperm DNA fragmentation by five different assays: TUNEL assay, SCSA, SCD test and alkaline and neutral Comet assay. Andrology 1: 715-722, 2013.

38. Huang CC, Lin DP, Tsao HM, Cheng TC, Liu CH and Lee MS: Sperm DNA fragmentation negatively correlates with velocity and fertilization rates but might not affect pregnancy rates. Fertil Steril 84: 130-140, 2005.

39. Mahfouz R, Sharma R, Thiyagarajan A, Kale V, Gupta S, Sabanegh E and Agarwal A: Semen characteristics and sperm DNA fragmentation in infertile men with low and high levels of seminal reactive oxygen species. Fertil Steril 94: 2141-2146, 2010.

40. Tran M, Uriondo H, Nodar F and Sedó CA: Cryopreservation promotes sperm DNA damage through oxidative stress $(38 \mathrm{~N})$. Obstetr Gynecol 131 (Suppl): 162S, 2018.

41. Zheng JJ, Chen X, Zhang LY, et al: Protective effects of vitamin $E$ and B12 on viability and DNA of frozen sperm. J Reproductive Med 27: 264-268, 2016.

42. O'Connell M, Mcclure N, Lewis SEM: The effect of cryopreservation on sperm morphology, motility and mitochondrial function. Hum Reprod 17: 704-709, 2002.

43. Liu L, Lao W, Ji QS, Yang ZH, Yu GC and Zhong JX Lycium barbarum polysaccharides protected human retinal pigment epithelial cells against oxidative stress-induced apoptosis. Int J Ophthalmol 8: 11-16, 2015.
44. Zhang WJ, Yang H, Zhu L, Luo Y, Nie L and Li G: Role of EGFR/ErbB2 and PI3K/AKT/e-NOS in Lycium barbarum polysaccharides ameliorating endothelial dysfunction induced by oxidative stress. Am J Chin Med 47: 1523-1539, 2019.

45. Li XM, Ma YL, Liu XJ: Effect of the Lycium barbarum polysaccharides on age-related oxidative stress in aged mice. J Ethnopharmacol 111: 504-511, 2007.

46. Taheri Moghadam M, Asadi Fard Y, Saki G and Nikbakht R Effect of vitamin $D$ on apoptotic marker, reactive oxygen species and human sperm parameters during the process of cryopreservation. Iran J Basic Med Sci 22: 1036-1043, 2019.

47. Hezavehei M, Mohseni Kouchesfahani H, Shahverdi AH, Sharafi M, Hosseini Salekdeh GH and Eftekhari-Yazdi P: Induction of Sublethal oxidative stress on human sperm before cryopreservation: A time-dependent response in post-thawed sperm parameters. Cell J 20: 537-543, 2019.

48. Dai GC: Effects of Lycium barbarum polysaccharide on apoptosis inhibition and cryopreservation of Goat Spermatogonial stem cells. Northwest Agricultural Forestry Univ, 2019.

49. Ma XF: Effects of Lycium barbarum polysaccharide on apoptosis, gene expression and delayed rectifier potassium current of retinal ganglion cells induced by high glucose. J Hainan Med Coll: 23, 2017.

50. Huang H, P H, W Y, et al: Inhibitory effect of Lycium barbarum polysaccharide on apoptosis of bone marrow monocytes induced by ionizing radiation. Environmental Occupational Med 35: 67-71, 2018.

51. Li FZ, Wang YH, Wen F, et al: Effects of Laminaria polysaccharide and Lycium barbarum polysaccharide on cryopreservation of goat semen. Environmental Occupational Med 39: 48-52, 2018.

52. Muratori M, Tarozzi N, Carpentiero F, Danti S, Perrone FM, Cambi M, Casini A, Azzari C, Boni L, Maggi M, et al: Sperm selection with density gradient centrifugation and swim up: Effect on DNA fragmentation in viable spermatozoa. Sci Rep 9: 7492,2019

This work is licensed under a Creative Commons Attribution-NonCommercial-NoDerivatives 4.0 International (CC BY-NC-ND 4.0) License. 